\title{
Comparing outcomes: the KISS principle
}

When I write this editorial, it is a rainy Sunday afternoon in the middle of an almost lockdown. Plenty of time to enjoy the new papers that you will find in this journal.

When we discuss science and our experiences, we need a common language to talk about our outcomes. You will all recognize the (panel)discussions where obviously apples and pears were compared. In 2018 Hopkins et al. , using an e-Delphi process, proposed a Core Outcome Set for four key domains of CRS (CHROME) (1). We use the SNOT-22 to describe the quality of life of our patients and the Lund-Mackay score to describe a CTscan ${ }^{(2)}$. The beauty of the Lund-Mackay score is its simplicity. It can be easily taught and remembered and probably the simplicity is next to the excellent other properties an important reason why it is so widely used. In this issue of the Journal, Reitsma et al. propose The Amsterdam Classification of Completeness of Endoscopic Sinus Surgery (ACCESS): a new CT-based scoring system grading the extent of surgery. The interrater agreement of the ACCESS score was excellent, comparable to the Lund-

Mackay score ${ }^{(2)}$ and I hope it will be as useful and widely used. EPOS2020 ${ }^{(3)}$ proposes a new classification for CRS: the division of CRS into primary and secondary disease, deciding whether it is a local or diffuse problem and endotyping into type 2 or nontype 2 that has direct implications for treatment. The introduction of biologicals was an important reason to better classify
CRS but also different inflammatory endotypes clearly exhibit different prognoses ${ }^{(4)}$ and endotyping drives the extent of our surgical treatment ${ }^{(5)}$. In this issue, Steiner et al. propose IL-13 as a possible specific biomarker in nasal secretions of patients with N-ERD, which may allow to differentiate between CRSwNP with vs. without N-ERD and may possible lead to differentiation in treatment. Also in this issue, Lin et al. show that adding lowdose clarithromycin to oral corticosteroids as post-ESS therapy gives improved outcomes in patients with CRSwNP, especially those without tissue eosinophilia.

The year 2020 will be remembered in history as the year the world was stricken by COVID-19. To say it has a significant impact on our lives is an understatement. For our journal, the year 2020 meant a substantial increase in the number of papers we received. To accommodate more papers in our Journal, we ask authors whenever possible to condense their papers to letters to the editor. In that way we hope to include significantly more papers. In this issue you can find a number of very interesting letters. COVID-19 ruled out meeting all of you in Thessaloniki. We plan to have the ERS2021 in Thessaloniki, September 2630, 2021. I wish all readers a healthy 2021 and hopefully we can all meet in September in Thessaloniki.

\section{References}

1. Hopkins C, Hettige R, Soni-Jaiswal A, Lakhani R, Carrie S, Cervin A, et al. CHronic Rhinosinusitis Outcome MEasures (CHROME), developing a core outcome set for trials of interventions in chronic rhinosinusitis. Rhinology. 2018;56(1):22-32.

2. Lund VJ, Mackay IS. Staging in rhinosinusitus. Rhinology. 1993;31:183-4.

3. Fokkens WJ, Lund VJ, Hopkins C, Hellings PW, Kern R, Reitsma S, et al. European Position Paper on Rhinosinusitis and Nasal Polyps 2020. Rhinology. 2020;58(Suppl S29):1-464.

4. Wei B, Liu F, Zhang J, Liu Y, Du J, Liu S, et al. Multivariate analysis of inflammatory endotypes in recurrent nasal polyposis in a Chinese population. Rhinology. 2018;56(3):216-26.

5. Moreno-Luna R, Gonzalez-Garcia J, Maza-Solano $J M$, Molina-Fernandez E, Pinheiro-Neto CD, Del Cuvillo Bernal A, et al. Free nasal floor mucosal grafting after endoscopic total ethmoidectomy for severe nasal polyposis: a pilot study. Rhinology. 2019;57(3):219-24

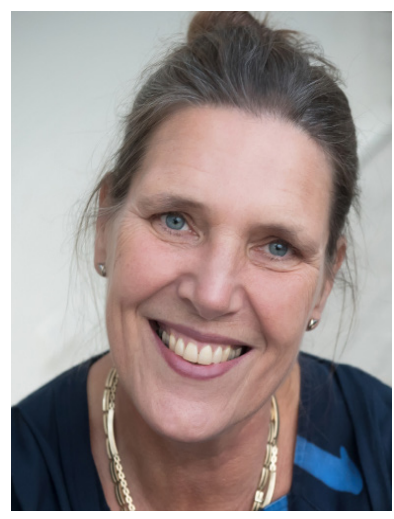

Wytske J. Fokkens, Editor-in Chief Amsterdam, the Netherlands 